\title{
Phasic alerting combined with visual spatial training: a novel therapeutic approach for unilateral spatial neglect
}

\begin{abstract}
Objective: Phasic alerting (PA) is a theory-motivated treatment approach for unilateral spatial neglect (USN). It aims to improve the non-spatial attentional components of the syndrome. The objective of this pilot study was to examine the potential benefit of a novel therapeutic approach, combining PA with Visual Spatial Training for individuals with USN.

Method: This pilot study, was conducted with 18 USN stroke patients allocated quasi-randomly to one of 3 groups (each 6 participants): experimental (receiving computerized Visual spatial Training combined with PA), 'spatial' control (receiving similar Visual spatial Training without PA), and 'non-spatial' control (receiving a similar amount of non-spatial cognitive treatment). Treatment comprised of 10 daily 30-minute sessions. Assessments of USN and function were made at 3 points in time: before and after the intervention period and after 5 weeks.
\end{abstract}

Results: The experimental group showed more than $50 \%$ amelioration in distinct measures of neglect during the treatment period. In contrast, the control groups did not show this trend during that period. All groups showed improvement at the follow-up period. However, in most tests the experimental group performed better with moderate effect size values in major USN tests.

Conclusion: Visual spatial training combined with PA shows promising results in producing amelioration in USN reflected both in measures of USN and ADLs.

Keywords: unilateral spatial neglect, rehabilitation, stroke, phasic alerting, visual search, spatial attention
Volume 3 Issue 3 - 2018

\author{
Asnat Bar-Haim Erez,' Nachum Soroker, ${ }^{2,3}$ \\ Noomi Katz \\ 'Research Institute for Health and Medical Professions, Ono \\ Academic College, Israel \\ ${ }^{2}$ Loewenstein Rehabilitation Hospital, Israel \\ ${ }^{3}$ Sackler Faculty of Medicine, Tel-Aviv University, Israel
}

Correspondence: Asnat Bar-Haim Erez, PhD, Faculty of Health Professions, The Occupational Therapy Department, Ono Academic College, 104 Zahal St, Kiryat Ono, 55000, Israe, Tel +972528631216, Email aaerez@gmail.com

Received: May 10, 2018 | Published: June 05, 2018
Abbreviations: PA, phasic alerting; USN, unilateral spatial neglect; RHS, right-hemisphere stroke; BIT: behavioral inattention test; MWCT, mesulam-weintraub random symbol cancellation task; FIM, functional independence measure

\section{Introduction}

Unilateral spatial neglect (USN) is a striking phenomenon in which a patient, usually after a right-hemisphere stroke (RHS), "appears to ignore the left side of space, as if that half of the world had abruptly ceased to exist in any meaningful form". ${ }^{1}$ The term describes the outcomes of damage to different brain structures and functions, commonly manifesting as a failure to attend, explore and respond correctly to objects or events in contralesional space. ${ }^{2,3}$ USN has been associated with poor recovery of functional skills and is one of the major factors impeding successful rehabilitation after stroke. ${ }^{4-6}$ It was shown that attention deficits may remain in these patients for long periods of time after the disappearance of more dramatic manifestations of USN. ${ }^{7}$

Various theories were formulated in an effort to explain USN, and other theory-driven approaches to treatment were suggested. One such approach, termed phasic alerting (PA), is based on accounts of the syndrome that stress the importance of deficient alertness or sustained attention. Phasic alertness represents the increased internal state of readiness to respond to an external warning stimulus and is believed to be critical in spatial selection tasks. ${ }^{8,9}$ Essentially, the treatment provides a randomly auditory stimulus that "alerts" the person. The anatomical substrate of this component of the attentional system is not clear. It was thought to be mediated by circuits involving the frontal cortex, possibly with the right hemisphere playing a dominant role. ${ }^{10}$ There is evidence suggesting that persistence of neglectrelated disability is determined in part by co-existence of damage to the non-spatially lateralized mechanisms involving the right inferior parietal and lateral frontal cortices. ${ }^{11,12}$ More recent research into the mechanism of phasic alerting in 6 USN patients, concluded that higher levels of alertness can help overcome the typical neglect symptoms, such as rightward lateralization and unilateral extinction. ${ }^{13}$

Robertson and colleagues ${ }^{8,9}$ conducted a series of experiments to study the possibility that the system involved in maintenance of alertness and the system mediating spatial attention interact in the formation of USN phenomena They have shown that PA can produce a beneficial effect on the ability of RHS patients with left USN to judge whether a visual event on the left did or did not precede the occurrence of a right sided event. The typical delay in perceiving left sided events improved significantly when a non-lateralized warning sound alerted patients phasically. The interaction between vigilance and orienting processes in USN was shown also by the fact that increased alertness produced by external cues reduced the magnitude of unilateral neglect. ${ }^{14}$ These studies led to the idea that PA may be used for alleviation of attention deficits in USN. ${ }^{15}$

The importance of evaluating the different elements of the attention network in the process of treatment planning for USN and of applying therapeutic exercises targeting defects of the different network 
components, was highlighted by various researchers. ${ }^{16,17}$ However, it remains largely uncertain what treatment is optimal for the individual patient, even when the specific pattern of structural brain damage and the specific combination of the various manifestations of impairment pertinent to that patient are known. In fact, the interactions described earlier make it difficult to assess the relative contribution of the different components. ${ }^{18}$

Research suggests that standard visual spatial training - a common treatment method used for treating USN in rehabilitationimproves perceptual scanning and is effective in treating visual neglect. However, its effectiveness in improving function is not conclusive. Furthermore, it was hypothesized that these results mirror improvements in the techniques in paper and pencil tasks, or in the clinic, but it does not transfer to everyday function..$^{18}$ Cicerone et al., ${ }^{19}$ in their evidence-based review of cognitive rehabilitation noted that when visual spatial training was combined with other techniques, such as complex visual scanning task or functional tasks, the improvement in functional outcome was significant.

Given the difficulties mentioned above in planning and understanding the specific elements that might assist in the successful rehabilitation of USN, we have designed a novel therapeutic approach to USN. This approach combines the PA principle with Visual spatial Training. The PA component was aimed to improve readiness to respond in general, i.e, the functioning of that part of the attention network that deals with alertness and maintenance of sustained attention and vigilance. ${ }^{9}$ The aim of the second component, the visual spatial training, was aimed at improving orienting and allocation of attention to contralesional stimuli. It comprised of feature and conjunction visual-search training was to improve the functioning of a different part of the attention network, dealing with orienting and selective allocation of attention to spatially-tagged target stimuli. The rationale for a combined approach stems from evidence suggesting: (1) that both elements are impaired in $\operatorname{USN}^{20}(2)$ that the element of alertness can benefit from exposure to warning stimuli; ${ }^{9}$ and (3) that the different parts of the attention network interact in the formation of USN symptomatology, ${ }^{21,22}$ as well as in the processes underlying recovery from this state. ${ }^{23}$

This pilot study was designed to examine the potential effect of this approach in treatment of individuals with USN post RCVA, by comparing it to spatial training alone and non-spatial training.

\section{Method}

\section{Design}

The experimental design for this study followed guidelines by Dobkin $^{24}$ who suggested a systematic guide for staging pilot studies. Our study fits the definition of a stage 2 pilot study, referred to as a 'development-of-concept trial', in which the purpose is to standardize a new intervention through the use of a control group and randomized group allocation.

\section{Participants}

All the individuals admitted to a Rehabilitation Hospital after stroke and met the inclusion criteria were recruited for this study. Inclusion criteria: first-event RHS; time after onset 2-6 weeks; age range: 40-75 years; right handedness; 6 years or more of formal schooling; stable clinical state; negative history of neurologic/psychiatric diseases; USN revealed on admission using standardized neglect tests (listed in the following section); willingness to participate confirmed by signing an informed consent. Individuals with hemianopsia, as determined by computerized visual field testing, were excluded from the study. 150 individuals with RHS were screened during a 2-year period. From these, 21 patients met the inclusion criteria and were included in the study. Two did not finish the follow-up stage (one passed away, unfortunately, and the second was discharged before the 5-week follow-up evaluation). At that point we had seven participants in one of the control groups, as opposed to six in the other two. Upon examining the severity of USN in each group, we observed that the patients in the group with seven members exhibited more severe symptoms. A decision was made to exclude one of the patients who showed extremely severe symptoms, and had reduced alertness that inhibited his participation in treatment. We felt that he would serve as an outlier, which would affect the results of the control group. Thus, the analyses of the study results included 18 patients, six from each of the 3 study groups. All participants remained in the inpatients rehabilitation center throughout the duration of the research. Table 1 describes patients' demographic, clinical and lesion data, the neurological data and impairment was done by the chief neurologist of the department. Figure 1 shows the flow of participants through the study. ${ }^{25}$

\section{Instruments}

Participants were diagnosed as having USN on the basis of deficits revealed in the next screening tests. This was done in order to relate both to an accepted test used to diagnose USN (BIT), as well to tests that are considered more sensitive (MWCT) and relate to the manifestations of USN in daily tasks (ADL checklist).

Behavioral Inattention Test (BIT) ${ }^{22}$ This is widely used battery for the assessment of neglect. The maximal score is 146 and the cut-off normative score is 130, The BIT cut-off scores were used to determine USN in this study. ${ }^{26}$

\section{Mesulam-weintraub random symbol cancellation task (MWCT) $)^{27,28}$}

Subjects are asked to identify a specific symbol printed 60 times (30 times on each side of the page) within a group of distracter stimuli of different shapes. A difference of 2 between the number of stimuli identified on the left and on the right is considered to be evidence of USN. We used the unstructured, more sensitive version.

\section{ADL checklist (catherine bergego scale) ${ }^{29,30}$}

Assessment of USN in the performance of ADL tasks. Ten items are rated on a 4-point (0-3) scale. Higher scores within the 0-30 scale indicate manifestations of more severe neglect in activities of daily living. A score of 1 is considered a sign of USN. Three additional assessments were also administered to the study participants in order to reflect the functional aspects of USN:

\section{Baking tray ${ }^{31}$}

Participants are asked to spread 16 cubes out evenly across a board "as if they were buns on a baking tray." The actual score indicates the number of buns placed on the left half of the tray; a score of 8 is considered to be normative. A rightward bias ( 7 cubes left $/ 9$ cubes right, or more) is considered an indication of left-sided neglect. Reported psychometric factors include $\mathrm{r}=.87$ for test-retest reliability. ${ }^{32}$ 
Table I Participants' demographic and clinical data

\begin{tabular}{|c|c|c|c|c|c|}
\hline \multirow{3}{*}{ Patient } & \multirow{3}{*}{ Age/ Sex } & \multirow{2}{*}{ Ed (yrs) } & \multicolumn{2}{|c|}{ Neurological impairment } & \multirow{2}{*}{ Lesion } \\
\hline & & & Motor & Sensory & \\
\hline & & \multicolumn{3}{|c|}{ Experimental group (combined treatment) } & \\
\hline AA & $49 / M$ & 8 & + & + & $\mathrm{MCA}(\mathrm{I})$ \\
\hline$A B$ & $62 / M$ & 8 & ++ & + & MCA (I) \\
\hline AC & $66 / M$ & 14 & ++ & ++ & MCA (I) \\
\hline AD & $75 / M$ & 15 & + & \pm & MCA (I) \\
\hline $\mathrm{AE}$ & $5 I / M$ & II & ++ & + & MCA (I) \\
\hline AF & $60 / M$ & 15 & \pm & \pm & $\operatorname{MCA}(\mathrm{H}$ \\
\hline Mean (SD) & $58.0(8.0)$ & II.7 (2.7) & + & + & MCA (I) \\
\hline
\end{tabular}

\section{Control group I -spatial treatment only}

\begin{tabular}{|c|c|c|c|c|c|}
\hline BA & $42 / M$ & 10 & ++ & ++ & $\operatorname{MCA}(\mathrm{I})$ \\
\hline BB & $62 / M$ & 14 & ++ & ++ & $\operatorname{MCA}(I)$ \\
\hline$B C$ & $52 / \mathrm{F}$ & 12 & ++ & + & MCA (I) \\
\hline $\mathrm{BD}$ & $64 / F$ & 14 & - & - & PCA $(H)$ \\
\hline $\mathrm{BE}$ & $59 / M$ & 12 & + & + & MCA (I) \\
\hline $\mathrm{BF}$ & $61 / M$ & 8 & + & \pm & MCA (I) \\
\hline \multirow[t]{2}{*}{ Mean (SD) } & $56.7(8.3)$ & \multicolumn{4}{|l|}{ II.3 (2.1) } \\
\hline & & \multicolumn{4}{|c|}{ Control group II-non-spatial treatment } \\
\hline $\mathrm{CA}$ & $54 / M$ & 8 & ++ & ++ & MCA (I) \\
\hline$C D$ & $66 / F$ & 9 & ++ & ++ & MCA (I) \\
\hline $\mathrm{CC}$ & $58 / M$ & 14 & ++ & ++ & $\operatorname{MCA}(\mathrm{I})$ \\
\hline $\mathrm{CE}$ & $44 / F$ & 12 & ++ & ++ & MCA (I) \\
\hline $\mathrm{CY}$ & $6 I / M$ & 15 & \pm & ++ & MCA (I) \\
\hline $\mathrm{CH}$ & $63 / M$ & 12 & \pm & ++ & $\operatorname{MCA}(\mathrm{H})$ \\
\hline Mean (SD) & $60.5(9.6)$ & II.5 (3.8) & 32.2 & & \\
\hline
\end{tabular}

Time since stroke onset (days): Experiment (mean 25.0, sd 9.8); Spatial (mean 3I.5, sd 6.3); Non-spatial (mean 3 I.2 sd I0.8). (Abbreviations: Ed, education; Impairment, $-/+/+/++=$ no/mild/moderate/severe, respectively, MCA, ACA, PCA=Middle; Anterior and Posterior cerebral arteries, I, ischemic infarction; $\mathrm{H}$, intracerebral haemorrhage). 


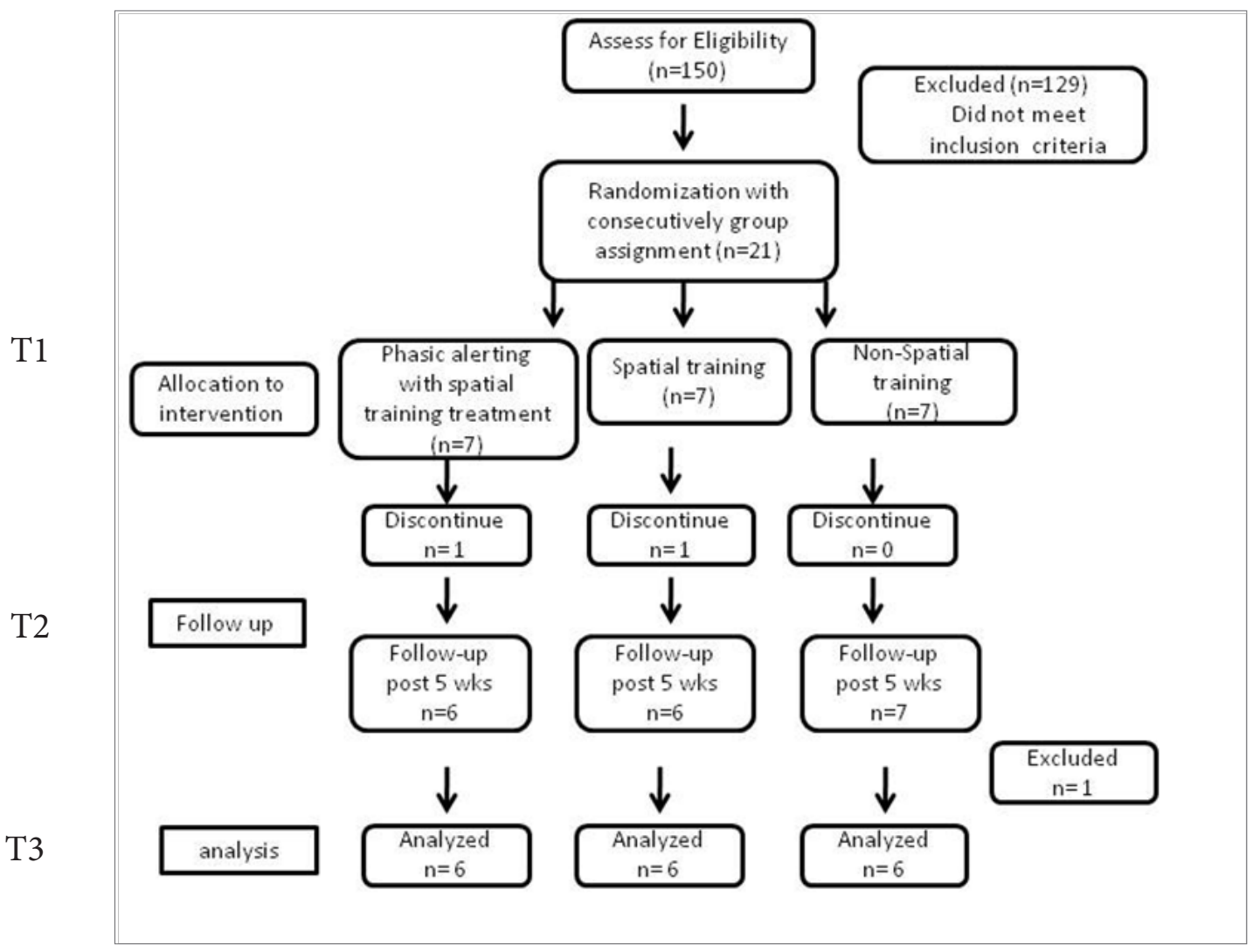

Figure I Flow of participants.

\section{Room Description ${ }^{33}$}

Subjects were seated in the center of a small room $(2.5$ by $3.5 \mathrm{~m})$ with their backs against an empty wall. We placed 6 objects on a table in front of them,( 3 on each side), i.e., a computer screen, a bottle of water, and a cup with pencils and pens. The patients were asked to identify 4 more items (a door, a window, a chair, a picture) aligned along both the left and right walls of the room. They were asked to name all the objects they see in the room within a period of 2 minutes. The number of items identified on each side was recorded.

\section{Functional Independence Measure (FIM) ${ }^{34}$}

The FIM is widely used to assess the degree of disability and burden of care in basic activities of daily living. It consists of 18 items that are rated on a 7-point ordinal scale, from 1 (indicating complete dependency) to 7 (indicating complete independence). The 18 items are divided into 2 factor scores: 13 items comprising the motor scale (maximum score 91) and 5 items comprising the cognitive scale (maximum score 35). Due to the variability in the participants' motor capabilities we used the cognitive scale as an outcome measure in this study.

All six tests described above were used to assess the individuals' progress and USN status.

\section{Procedure}

This study design was fashioned after the study of Frassinetti et al., ${ }^{33}$ in which the effectiveness of prism adaptation in USN was examined. Our pilot study was designed to examine a novel intervention with new strategies for the treatment of USN. In this study, one of the researchers randomly assigned patients with USN into one of 3 groups. As the number of USN patients was not large and the number assigned to each group was to be equal, the following quasi-randomization method was used to form the groups. The first individual was assigned randomly to one of the groups (each group was given a number from 1-3, and similarly, a number from 1-3 was drawn for that patient). From that point on each new participant was assigned to the next group consecutively.

Evaluation was conducted at 3 time points: Pre-intervention T1: participants with documented USN based on the BIT, MWCT and ADL Checklist went through the additional 3 assessments of USN and function described above; Post- intervention T2: following the 2-week treatment phase, a second evaluation was conducted to assess progress. Patients then continued the rehabilitation process for 5 more weeks according to their individual needs, using the conventional treatment of the occupational therapy department (i.e., training leftward scanning and increased awareness). Follow-up T3: after the 5 additional weeks of the above treatment, the testing was repeated. All the evaluations were done by the primary investigator, using standardizes tests. At T2 and T3 the researcher was not blind to the participant's group allocation but was not involved in the treatment process. Only standardized tests were used to avoid this bias. By the end of the data-collection period, analysis of statistical power ${ }^{35}$ indicated a power of $60 \%$ at $\alpha=0.05$ with the current sample size of $\mathrm{n}=6$ in each group.

Treatment with the targeted intervention included 10 daily 
30-minute sessions (Sunday-Thursday) over a 2-week period (experimental/control). Each group was treated by a different occupational therapist from the stroke-unit department who was not part of the researchers' team. Each therapist was assigned to a treatment group and training was provided by the main researcher The chief occupational therapist and the supervisor for the stroke unit were involved in the training and supervision of the research process. Participants remained in the in-patient rehabilitation department throughout the research period.

\section{Experimental group}

Participants in this group received a combined visual spatial attention training incorporating PA principles. The visual spatial attention training was provided through a computerized program for the assessment and training of visual spatial attention (VISSTA) ${ }^{36}$ Only the training component of the VISSTA was used. The program addresses two levels of attentional demand in search tasks, based on Triesman's feature integration theory. ${ }^{37}$ Subjects are asked to detect a visual target presented in each trial at a different part of the screen. The target stimulus is surrounded by distracter stimuli. In the easier (popout; feature search) condition, the target differs from all distracters by a single feature - color. In the more demanding condition (conjunction search) it is distinguished by a specific combination color and shape. The PA principles component of the treatment was accomplished by sounding of a loud pitch tone emitted every 15,30 or 45 seconds at random from a buzzer (that was designed for this research) placed in front of the computer in mid-sagittal plane. Patients were told not to respond to the tone but to continue with their task. No mediation cues were given by the therapist during the training, but feedback concerning task performance was given at the end of the session.

\section{First control group-spatial training}

Participants received the same visual spatial attention treatment with the VISSTA program as the experimental group, but without using the PA component. The aim was to assess the added value of PA, beyond the visual spatial training.

\section{Second control group- non-spatial training}

Participants in this group received daily cognitive treatment that did not include visual spatial training, for a similar period of time as the other groups. The treatment also did not include the standard visual spatial search methods, but included mainly executive function training (such as planning and problem solving exercises) and verbal memory training, in accordance with the patient's needs.

In addition, all subjects received daily sessions of 30 minutes in the occupational therapy department, concentrating on motor activation to improve upper extremity function, and treatment targeting improvement in activities of daily living. Participants did not get any additional treatment aiming at improving visual spatial orientation. All the participants also received daily physiotherapy treatment. Assessments of treatment effect were made by one of the authors, who were blind to the subjects' group allocation.

\section{Statistical Analysis}

Initially statistics included analysis of variance between groups (ANOVA) on the average score of the achievement of potential improvement. Due to the small sample size, the variability in neglect severity within and between patient groups and the aim of this pilot study, to examine potential benefit for the treatment, we carried to specific analyses: (a) achievement of potential improvement; (b) calculated effect size (d) to assist us to interpret the trend in amelioration of USN we observed during the study period.

The achievement of potential improvement reflects the proportion of potential improvement achieved during rehabilitation It is also termed relative improvement and has been used as a quantitative measure of recovery of function used in diverse populations. ${ }^{38-40} \mathrm{It}$ is expressed as the actual improvement divided by the potential for improvement in a given measure. ${ }^{41}$ The equation is calculated individually for each patient in the following way: post-treatment score minus pre-treatment score divided by the difference between the maximal score and the pre-treatment score. This measure provides a value from 0 to 1 , where 0 represents no improvement between the two assessments and a score of 1 represents the maximal progress that could be made. The achievement of potential improvement was calculated separately for each patient and then averaged for each group.

The research was approved by the Human Rights (Helsinki) Committee and the Health Ministry, and all the participants signed an informed consent form before entering the study.

\section{Results}

\section{Achievement of potential improvement}

We compared the achievement of potential improvement by each group, as explained in the Methods section. The results (Table 2) (Figure 2) point to a distinct time course in the achievement of potential improvement by each group. The experimental group achieved more than $50 \%$ of its average potential for improvement during the twoweek intervention period (T1-T2) in all measures except for the FIM. In contrast, the achievement of potential improvement during that time, by the non-spatial control group, was smaller than $50 \%$ in all measures. In the spatial control group, the improvement during the treatment period was smaller than $50 \%$ in all measures except for the Room Description and the Baking Tray.

The three groups made further amelioration in all tests during the subsequent 5 weeks of follow-up, during which time all groups received the standard physiotherapy and occupational therapy treatments given in the rehabilitation center, including training to orient to left and increased awareness to functional deficits due to USN. Statistical analyses, ANOVA, of these differences were not significant. We calculated the effect size (Cohen's d) to better understand the trend of achievement of potential improvement that we observed. The d values suggest that in the post two-week intervention period (T1-T2) there was strong effect for the change measure by the BIT; and moderate effect in the WMCT, room description and the ADL checklist. Indication that the change observed between the experimental group and the control groups might has significance. Weaker effects were found in the change in the baking tray and the FIM measures. Examination of effect size for the whole research period (T1-T3) revealed lower effect size values except for the WMCT and ADL checklist, which indicated moderate effect size (Table 2).

The measures' raw scores for the three groups, across time, can be viewed in Figure 3. One can view the same trend, described in the previous section, however due to the variability in baseline measures; analysis was conducted on the relative change. 
Table 2 Relative improvement data with effect size

\begin{tabular}{lllllllll}
\hline Measure & \multicolumn{2}{l}{ TI-T2 Mean (SD) } & & Effect size & TI-T3 Mean (SD) & Effect size \\
\cline { 2 - 8 } & Experiment & Spatial & Non-spatial & $d^{*}$ & Experiment & Spatial & Non-spatial & $d^{*}$ \\
\hline BIT & $0.60(0.24)$ & $0.31(0.29)$ & $0.30(0.28)$ & 0.9 & $0.79(0.13)$ & $0.56(0.40)$ & $0.65(0.28)$ & 0.4 \\
WMCT & $0.55(0.23)$ & $0.25(0.5)$ & $0.19(0.35)$ & 0.5 & $0.79(0.16)$ & $0.59(0.36)$ & $0.49(0.25)$ & 0.6 \\
ADL & $0.59(0.32)$ & $0.25(0.46)$ & $0.16(0.21)$ & 0.6 & $0.59(0.35)$ & $0.21(0.4 I)$ & $0.41(0.12)$ & 0.6 \\
FIM & $0.46(0.35)$ & $0.22(0.14)$ & $0.18(0.29)$ & 0.4 & $0.57(0.29)$ & $0.38(0.45)$ & $0.48(0.15)$ & 0.3 \\
Room Descript. & $0.96(0.4)$ & $0.60(0.37)$ & $0.10(0.58)$ & 0.7 & $0.83(0.41)$ & $0.59(0.39)$ & $0.64(0.47)$ & 0.3 \\
Baking Tray & $0.56) 0.44)$ & $0.72(0.7)$ & $0.25(0.39)$ & 0.4 & $0.62(0.49)$ & $0.80(0.39)$ & $0.56(0.55)$ & 0.3
\end{tabular}

*effect size cohen's d;TI, assessments pre-intervention; T2, assessments immediately post intervention; T3, assessments at follow- up (5 weeks post intervention)
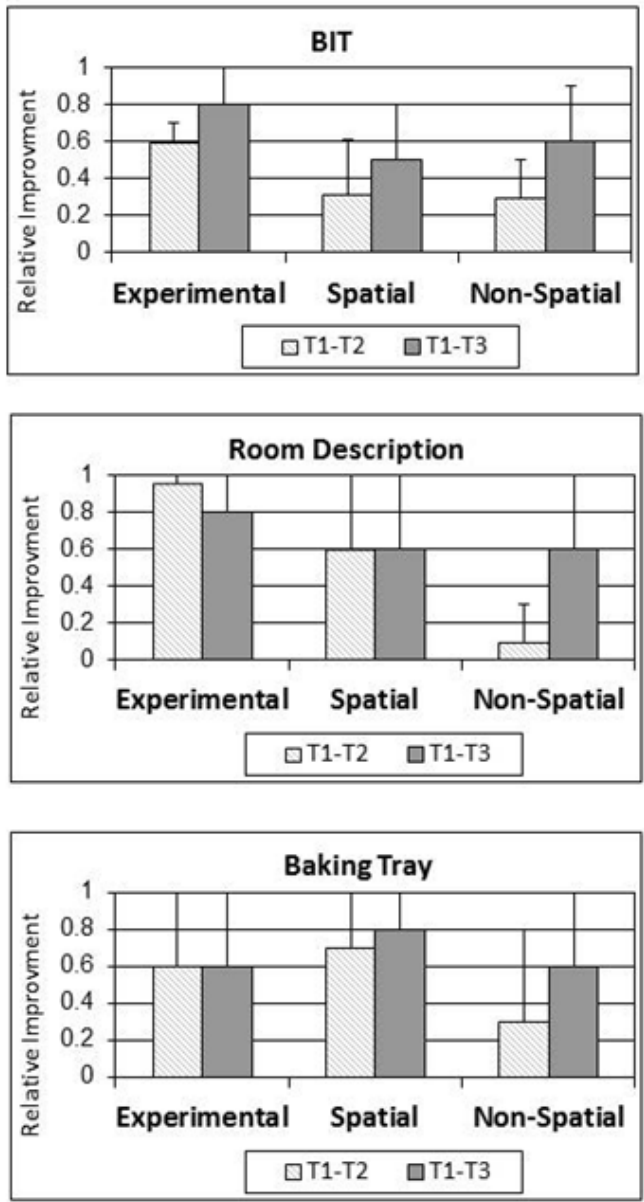
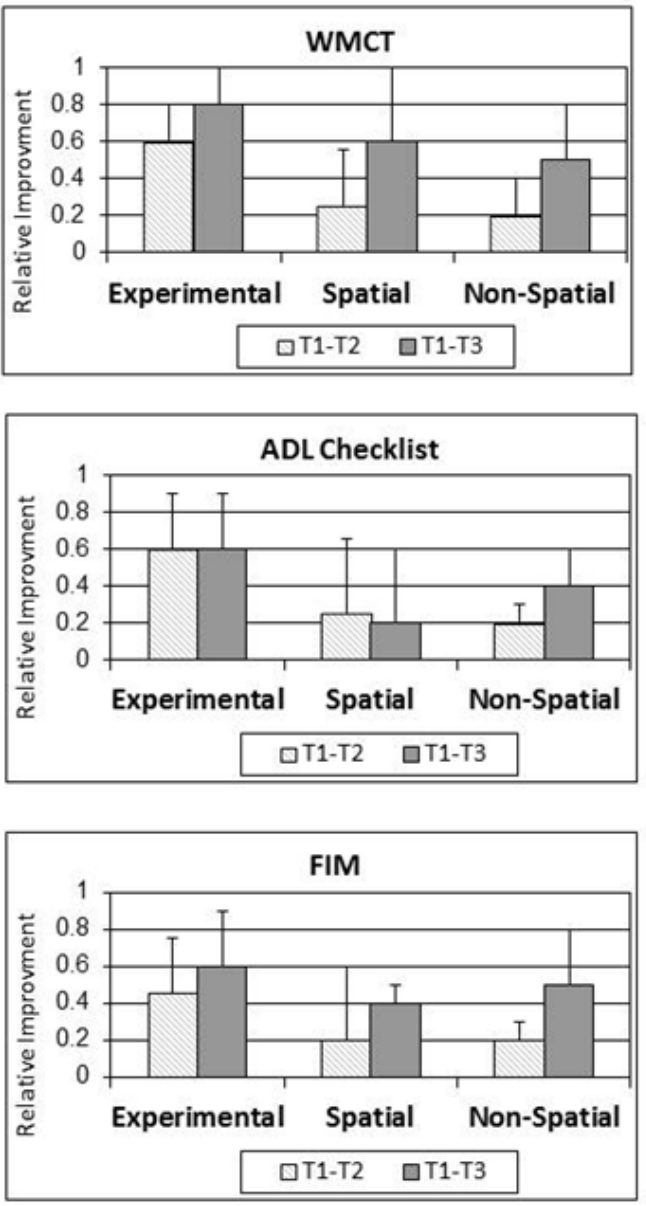

Figure 2 Achievement of potential improvement: group comparison in various tests.

Mean (and standard deviation) group values of improvement accomplished during the intervention period (TI-T2; 2 weeks) and during the entire research period (TI-T3; 7 weeks); Effect size data is for the entire period (TI-T3). (Abbreviations: Exp, experimental group; Control I, First ('spatial') control group; Control II, Second ('non-spatial') control group).

Citation: Erez AB, Soroker N, Katz N. Phasic alerting combined with visual spatial training: a novel therapeutic approach for unilateral spatial neglect. Int Phys Med Rehab J. 2018;3(3):243-25I. DOI: I0.15406/ipmrj.20I8.03.00 I I 


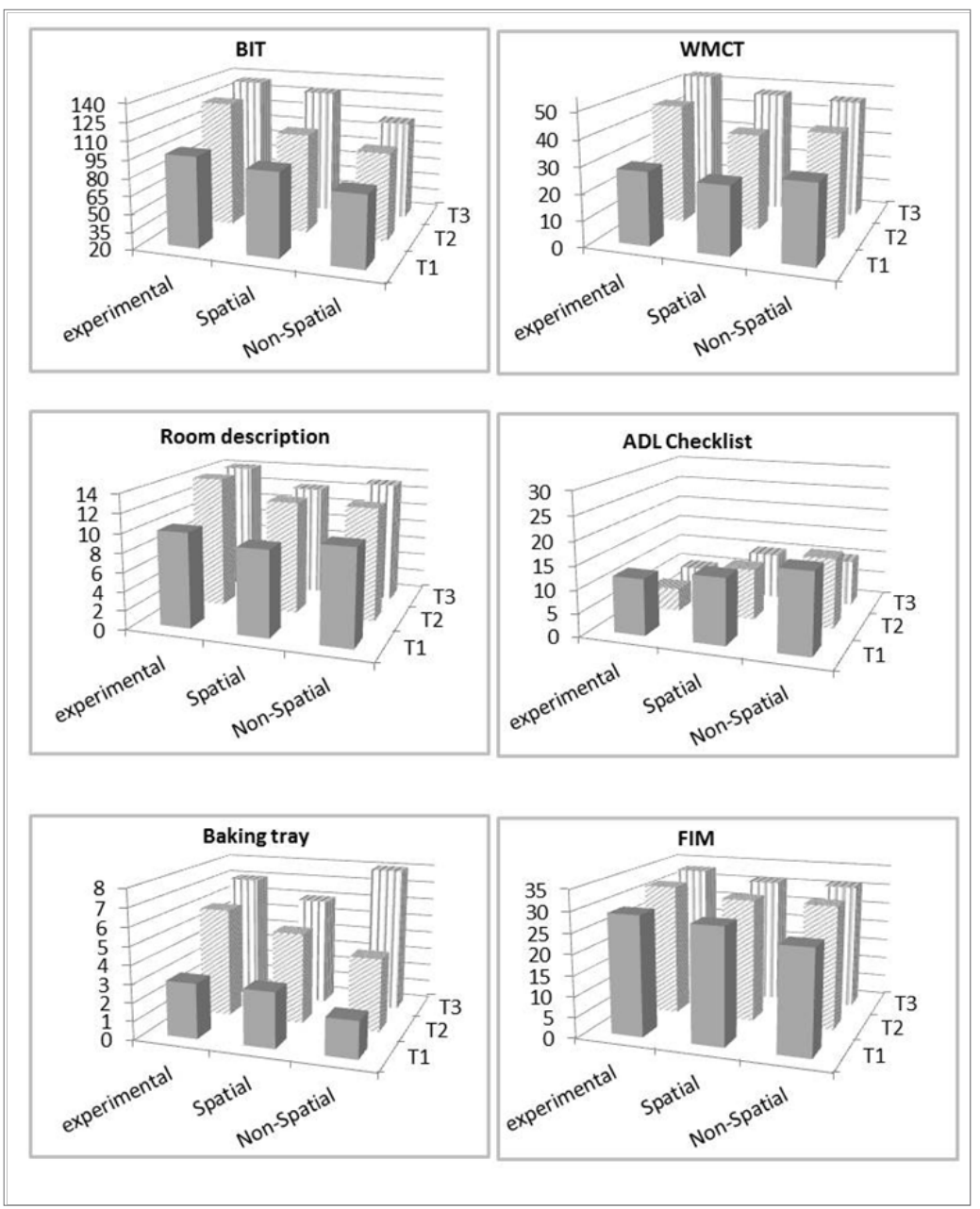

Figure 3 Performance on the various measurements:Across time and groups.

*Y axis reflects total maximum score for that measure.

**ADL Checklist score- Higher scores indicate manifestations of more severe neglect in activities of daily living.

\section{Discussion}

Our objective was to examine the potential benefit of a novel therapeutic approach combining phasic alerting (PA) with visual spatial training, for individuals with USN.

Eighteen right-hemisphere damaged patients with left USN, $4 \pm 2$ weeks after stroke onset, were allocated quasi-randomly to one of 3 groups. In the experimental group patients received 10 daily sessions comprised of computerized visual search task combined with PA. In the 'spatial' control group patients received similar Visual spatial training without the PA component, and in the 'non-spatial' control group patients received a similar amount of cognitive training unrelated to spatial attention. Assessments of USN severity (using 5 different standardized measures) and of overall functional capacity in ADL (using the FIM as a measure), were made immediately before and after the 2-week treatment period, and after an additional 5-weeks of follow-up, in which all patients received standard occupational and physical therapy treatment in accord with their needs.

Due to the small sample and variability in USN among the participants analysis included examination of the average 'achievement of potential improvement' in each group and effect size values between the groups. This analysis suggests that the experimental group, but not the two control groups, achieved more than $50 \%$ of the potential for improvement in the different measures, during the 2 weeks of treatment, with moderate to strong effect size. The improvement in the two control groups was more attenuated and delayed and appeared to occur mainly at the second stage after receiving standard treatment that included also visual spatial training. The measures that were sensitive to the differences, post treatment, were the paper and pencil measures and two of the functional measures of USN. At the follow up these differences decreased as marked ameliorations were made by the other control groups. However, examination of the differences and effect size for the overall research time (T1-T3) revealed that in two measures (WMCT and the ADL checklist) the PA group had advantage over the other two groups, i.e., they showed amelioration in USN symptoms to a greater extent. These measures are considered sensitive to change and reflect different measures for USN, neurobehavioral vs. observation of function in ADL.

Thus, the treatment comprised of only 10 daily sessions of visual spatial training combined with PA, produces amelioration in USN at 
the acute and sub acute phases post stroke, both in spatial attention and in its manifestations in daily activities. This combined treatment has an advantage compared to cognitive intervention that does not specifically address problems of spatial attention. Comparison to the group that received visual spatial training without the PA component yielded more modest results at the end of research period. Differences were apparent immediately post treatment in major neurobehavioral and functional tests and to a lesser extent at the end of the research, attesting to the added value of the latter especially in the early stages of rehabilitation. These results corroborate earlier findings reported by Robertson and colleagues, suggesting that USN manifestations are likely to ameliorate following training using PA. ${ }^{9,15}$

Teasell et al., ${ }^{42}$ suggested that rehabilitation interventions for USN may be classified into two types: (a) those that attempt to increase patient's awareness, or attention, to the neglected space, and which include visual scanning retraining, and feedback to increase awareness of neglect; and (b) interventions that attempt to improve USN by targeting deficits associated with position sense and spatial representation, such as, prisms adaptation and eye-patching. In the current study two techniques aiming at increasing awareness and attention were combined to enhance their effect - visual spatial retraining and activation strategies.

Visual spatial training is a common practice in occupational therapy treatment for USN. Research suggests that this practice is effective, however, it is not clear whether the effects are sustained over time and whether there is a transfer to everyday function. ${ }^{19}$ In general, results of the current pilot study indicated that participants who received just computerized visual spatial training showed only modest decline in USN symptoms, however, benefited from treatment that addressed spatial and functional issues. Post the two weeks of initial training all groups received the standard occupational therapy treatment, for 5 weeks, that included- visual spatial training, increased awareness to deficits and their functional implications, and transfer of strategy and skills to everyday life. At that stage the two control groups showed improvement in USN symptoms, in their neurobehavioral and functional test results. This also implies that just training to detect stimuli on left is not sufficient and multi methods should be used. Still, current study results, lends support for the use of other methods as well, at least at the acute- post acute stages. This means adding to the standard visual spatial training methods that are based on neuroanatomical theories, such as PA.

\section{Study limitations}

Results should be treated with caution due to several study limitations. The sample size in each group was small (although generally compatible with other treatment studies in the neglect literature).$^{33}$ Given the multi-factorial nature of USN, the combination of small sample sizes and large inter-personal variance in USN severity and sensorimotor status, make it difficult to reach clear-cut generalized conclusions with regard to treatment efficacy for the varied gamut of neglect-related disturbances. The variability was apparent in the BIT and ADL scores, which were different between groups at baseline (Figure 3). Although the differences were not statistically significant, this may had an impact on the results. Another effect on results may be the omission of one participant with of severe USN in efforts to equate groups and exclude outliers. Following previous studies [9; 33 ] the design of the present research provided patients with only 10 designated treatment sessions. This raises a question as to whether a longer treatment period would have provided better or clearer results. The promising results obtained in our study encourage further study using a longer period of intervention.

\section{Future research}

Despite its limitations, the current pilot study yielded promising results that can lead to the stage of planning a well-designed RCT for treatment of USN using PA principles. The next study to examine this concept of intervention should take into account the following methodological issues (based on Dobkin ${ }^{24}$ ): 1) Sample size should be significantly higher to assess the intervention efficiency between combined treatment and spatial treatment alone. In this study, the potential effect size was moderate, indicating that a sample size of 6 per group is much less than necessary to prevent errors; (2) Analysis of treatment efficiency should take into account the USN severity as well as the USN types (i.e, personal/extra-personal dimensions); and 3) It could be useful to include a measure for level of alertness, or attentiveness, as the purpose of the PA intervention is to stimulate this system. Thus, a direct measure can shed more light on the PA intervention.

\section{Conclusion}

The findings, of this pilot study, suggest that phasic alerting (a cognitive neuroanatomical based treatment model) used in conjunction with computerized Visual spatial training at the early subacute stage has a positive effect in the rehabilitation of individuals with unilateral spatial neglect. The improvement in USN symptoms was maintained at least up to 2-months post treatment. Further RCT studies are needed to substantiate evidence based practice for the rehabilitation of USN. In this study we used various assessment methods- neurobehavioral and functional tests. This gave us a wide understanding on the nature of USN and its effect on function. It is also apparent that besides the BIT the WMCT the functional assessment measures, such as the ADL checklists and the baking tray, can provide with ecological sensitive information. We encourage clinician, in the neurological rehabilitation field, be involved in examination of various treatment techniques, such as, PA, prism adaptation, eye patching, and more; to examine their effect on function as well as mapping the best treatment to the specific severity or type of USN.

\section{Acknowledgements}

We would like to thank Lowenstein's professional and management staff and in particular the Occupational Therapy services for supporting and assisting in conducting this importnat and complex research. In specific, we would like to thank Mrs. Sara Averbuch (the former head of the occupational therapy services ) and Mrs. Dorit Hepner (the former supervisor of the CVA department) for their invaluable clinical support and insight which ensured the successful completion of the research

\section{Conflict of interest}

The author reports no conflicts of interest. The authors alone are responsible for the content and writing of the paper. No party has a direct interest, neither a financial relationship nor will they be conferred any benefits from writing this submission or the results of this research.

\section{References}

1. Halligan PW, Marshall JC. The history and clinical presentation of neglect. In: Robertson IH, Marshall JC, editors. Unilateral neglect: clinical and experimental studies. Hillsdale, NJ: Lawrence Erlbaum Associates; 1993:3-25. 
2. Barrett AM, Buxbaum LJ, Coslett HB, et al.Cognitive rehabilitation interventions for neglect and related disorders: moving from bench to bedside in stroke patients. $J$ Cog Neurosci. 2006;18:1223-36.

3. Mesulam M. A cortical network for directed attention and unilateral neglect. Ann Neurol. 1981;10(4):309-25.

4. Gillen R, Tennen H, McKee T. Unilateral spatial neglect: Relation to rehabilitation outcomes in patients with right hemisphere stroke. Arch Phys Med Rehabil. 2005;86(4):763-767.

5. Katz N, Hartman MA, Ring $\mathrm{H}$, et al. Functional disability and rehabilitation outcome in right hemisphere damaged patients with and without unilateral spatial neglect. Arch Phys Med Rehabil. 1999;80(4):379-84.

6. Monaco MD, Schintu S, Dotta M, et al. Severity of unilateral spatial neglect is an independent predictor of functional outcome after acute inpatient. Arch of Phys Med Rehab. 2011;92:1250-1256.

7. Robertson JH. The relationship between lateralized and non-lateralized attentional deficits in unilateral neglect. In: Robertson IH, Marshal JC, editors. Unilateral neglect: clinical and experimental studies. Hillsdale, NJ: Lawrence Erlbaum Publishers; 1993;257-75.

8. Robertson IH, Manly $\mathrm{T}$, Beschin N, et al. Auditory sustained attention is a marker of unilateral spatial neglect. Neuropsychologia 1997;35(12):1527-32.

9. Robertson IH, Mattingley JB, Rorden C, et al. Phasic alerting of neglect patients overcomes their spatial deficit in visual awareness. Nature. 1998;395:169-72.

10. Posner MI, Petersen SE. The attention system of the human brain. Annu Rev Neurosci. 1990;13:25-42.

11. Husain M, Rorden C. Non-spatially lateralized mechanisms in hemispatial neglect. Nature Reviews Neuroscience. 2003;4:26-36.

12. Sturm W, Longoni F, Weis S, et al. Functional reorganization in patients with right hemisphere stroke after training of alertness: a longitudinal PET and fMRI study in eight cases. Neuropsychologia. 2004;42:434 450.

13. Finkea K, Matthias E, Keller I, et al. How does phasic alerting improve performance in patients with unilateral neglect? A spatial weighting mechanisms systematic analysis of attentional processing capacity. Neuropsychologia. 2012;50(6):1178-1189.

14. Robertson IH, Tegner R, Tham K, et al. Sustained attention training for unilateral neglect: theoretical and rehabilitation implications. J Clin Exp Neuropsychol. 1995;17:416-30.

15. Robertson IE. Do we need the "lateral" in unilateral neglect? Spatially nonselective attention deficit in unilateral neglect and their implications for rehabilitation. Neuroimage. 2001;14(1):S85-S90.

16. Pierce SR, Buxbaum LJ. Treatment of unilateral neglect: a review. Arch Phys Med Rehabil. 2002;83(2):256-67.

17. Pizzamiglio L, Guarriglia C, Antonucci G, et al. Development of a rehabilitative program for unilateral neglect. Restor Neurol Neurosci. 2006;24(4-6):337-345.

18. Bowen A, Lincoln NB. Cognitive rehabilitation for spatial neglect following stroke. Cochrane Database of Systematic Reviews. 2013;(7):CD003586.

19. Cicerone KD, Dahlberg C, Malec JF, et al. Evidence-based cognitive rehabilitation: updated review of the literature from 1998 through 2002 Arch Phys Med Rehabil. 2005;86:1681-92.

20. Posner MI, Raichle ME. Networks of attention. In: Posner MI, Raichle ME, editors. Images of the Mind. New York: Scientific American Library; 1997:153-79.
21. Fan $\mathrm{J}, \mathrm{Gu} X$, Guise $\mathrm{KG}$, et al.Testing the behavioral interaction and integration of attentional networks. Brain Cog. 2009;70(2): 209-220.

22. Wilson BA, Cockburn J, Halligan PW. The development of a behavioral test of visuospatial neglect. Arch Phys Med Rehabil. 1987;68(2):98-102.

23. Corbetta M, Kincade MJ, Lewis C, et al. Neural basis and recovery of spatial attention deficits in spatial neglect. Nature Neurosci. 8(11):16031610.

24. Dobkin BH. Progressive staging of pilot studies to improve phase III trials for motor interventions. Neurorehabil Neural Repair. 23(3):197203.

25. Altman DG, Schulz KF, Moher D, et al. The Revised CONSORT Statement for Reporting Randomized Trials: Explanation and Elaboration. Ann Intern Med. 2001;134(8):663-694.

26. Halligan PW, Cockburn J, Wilson B. The behavioral assessment of unilateral neglect. Neuropsychol Rehabil. 1991;84(1):5-32.

27. Weintraub S, Mesulam MM. Right cerebral dominance in spatial attention. Further evidence based on ipsilateral neglect. Arch Neurol. 1987;44(6):621-5

28. Weintraub S, Mesulam MM. Visual hemispatial inattention: stimulus parameters and exploratory strategies. J Neurol Neurosurg Psychiatry. 1988;51(12):1481-8.

29. Azouvi P, Marchal F, Samuel C, et al. Functional consequences and awareness of unilateral neglect: study of an evaluation scale. Neuropsychol Rehabil. 1996;6(2):133-50.

30. Azouvi P, Olivier S, Montety G, et al. Behavioral assessment of unilateral neglect: study of the psychometric properties of the Catherine Bergego Scale. Arch Phys Med Rehabil. 2003;84:51-7.

31. Tham K, Tegner R. The baking tray task: a test of spatial neglect. Neuropsychol Rehabil. 1996;6:19-25.

32. Bailey M J, Riddoch MJ, Crome P. Test-retest stability of three tests for unilateral visual neglect in patients with stroke: star cancellation, line bisection and the baking tray task. Neuropsychol Rehabil. 2004;14(4):403-19.

33. Frassinetti F, Angeli V, Meneghello F, et al. Long-lasting amelioration of visuospatial neglect by prism adaptation. Brain. 2002;125(3):608-23.

34. Granger CV, Cotter AC, Hamilton BB, et al. Functional assessment scales: a study of persons after stroke. Arch Phys Med Rehabil. 1993;74(2):133-8.

35. Cohen J. A power primer. Psychol Bull. 1992;112(1):155-9.

36. Erez AB, Katz N, et al. Assessment of spatial neglect using computerized feature and conjunction visual search tasks. Neuropsychol Rehabil. 2009;19(5):677-95.

37. Treisman A. Gelade G. A feature integration theory of attention. Cog Psychol. 1980;12:97-136.

38. Paolucci S, Antonucci G, Grasso MG, et al. The role of unilateral spatial neglect in rehabilitation of right brain-damageed ischemic stroke patients: a matched comparison. Arch Phys Med Rehabil. 2001;82(6):743-9.

39. Katz N, Keren N. Effectiveness of occupational goal intervention for clients with schizophrenia. Am J of Occ Ther. 2011;65(3): 287-296.

40. Rand D, Katz N, Weiss PL. Intervention using the VMall for improving motor and functional ability of the upper extremity in post stroke. Eur $J$ Phys Rehabil Med. 2009;45(1):113-21.

41. Shah S, Vanclay F, Cooper B. Efficiency, effectiveness, and duration of stroke rehabilitation. Stroke. 1990;21(2):241-6.

42. Teasell, R, Salter K, Bitensky J, et al. Perceptual Disorders. EvidenceBased Review of Stroke Rehabilitation. 2011 